\title{
A simulation tool to predict the impact of soil topologies on coupling between a light rail system and buried third party infrastructure
}

\author{
C. Charalambous, Member, IEEE, I. Cotton, Member, IEEE, P Aylott
}

\begin{abstract}
The production of stray currents by DC Light Rail Systems leads to the corrosion of the supporting and third party infrastructure in close proximity to the rail system. This work simulates two parallel tracks that are occupied by two trains; one on each track. This type of modeling constitutes a realistic case study that is utilized to investigate the effect of soil topologies on the corrosion performance of a floating DC Light Rail System focusing on the supporting and third party infrastructure. The modeling technique used involves the accurate computation of the shunt and series parameters for use in a resistive type model using a commercially available software package. The results demonstrate the importance that soil resistivity has on the corrosion risk to traction system and third party infrastructure. Such information could ultimately be used to vary the level of stray current protection across a light rail system to ensure an consistent lifetime across the whole system.
\end{abstract}

Index Terms- Corrosion, DC light rail, soil topologies, stray current.

\section{INTRODUCTION}

$\mathrm{C}$ urrent leakage from DC railway systems is an inevitable consequence of the use of the running rails as a mechanical support / guide way and as the return circuit for the traction supply current. Since the rails have a finite longitudinal, or series, resistance - around $40-80 \mathrm{~m} \Omega / \mathrm{km}$ or $40-80 \mu \Omega / \mathrm{m}$ of rail - and a poor insulation from earth - typically from 2 to $100 \Omega \mathrm{km}$ - a proportion of the traction current returning along them will leak to earth and flow along parallel circuits (either directly through the soil or through buried conductors) before returning onto the rail and the negative terminal of the DC rectifier.

Given that current flow in a metallic conductor is electronic, whilst that through electrolytes such as the earth, concrete etc. is ionic, it follows that there must be an electron to ion transfer as current leaves the rails to earth. Where current leaves the rail to earth and an anode is produced there will therefore be an oxidation, or electron producing, reaction.

${ }^{1}$ Charalambos Charalambous and Ian Cotton are with the School of Electrical and Electronics Engineering, University of Manchester, M60 1QD, UK (e-mail: charalambos.charalambous@manchester.ac.uk). Pete Aylott is with CAPCIS, 1 Echo Street, Manchester, M1 7DP
Corrosion of metallic objects will therefore occur from each point that current transfers from a metallic conductor, such as a reinforcement bar in concrete, to the electrolyte (i.e. the concrete). Hence stray current leakage can cause corrosion damage to both the rails and any other surrounding metallic elements.

A key factor in determining the level of threat to the third party infrastructre (utilities) and the supporting infrastructure is the resistivity of the surrounding soil. Typically, a uniform soil resistivity model is assumed over the length of a system. In reality, it will change with both depth and system chainage.

This paper builds on previous work carried out by the authors [1], [2], [3]. It describes a simulation tool that can be used to predict the level of stray current (and hence corrosion damage) in a DC Light Rail System where the soil resistivity varies in the manner described above. In certain situations where the soil resistivity changes with depth, the buried utilities can experience significant protection from the stray current. However, a sudden change in soil resistivity along the length of the system can concentrate corrosion on a specific location. In such a case, protective measures may be specified at such a location to ensure the system lifetime is not compromised.

The simulation tool accurately calculates the current distribution between the rail, stray current collection mat and the buried utility. The modeling technique is demonstrated with a model of two parallel tracks that are occupied by two trains; one on each track. This type of modeling constitutes a realistic case study that can be utilized to investigate the stray current performance of a DC Light Rail System. Dynamic modeling as well as static modeling is possible with this technique.

Since there is a tendency in new transit systems to utilize floating running rails, literature stating that floating running rails are the best option if the stray current is to be minimized [3], [4] and [5]), this paper specifically addresses the corrosion performance of floating DC light rail systems. However, the simulation tool does have the capability to incorporate solidly 
bonded and diode-bonded systems [6].

\section{THE MODEL SYSTEM}

\section{A. Introduction}

The essential elements of a transit system are the rails, the rail-to-earth insulation, the power supply and the vehicles. The design and placement of these elements of the transit system dictates the stray current performance in terms of the total stray current leaving the rails [1], [3].

The work carried out in [1] described the design principles employed in CDEGS [7] to accurately model the current distribution between structures of a DC light rail system, by taking into consideration the geometry of the system, the soil topology and the interactions between the metallic structures under study. Figure 1 illustrates the perspective view of the CDEGS model, for the rails, their supporting infrastructure (trackbed) and a third party infrastructure (e.g. buried metallic pipe).

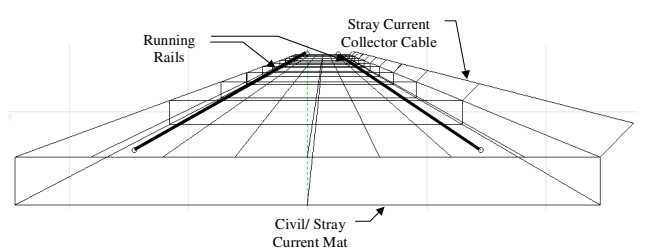

Fig. 1. Geometrically Accurate CDEGS model in perspective view

The work carried out in [2] described a simulation tool that is a two step process combining the accuracy of CDEGS with the flexibility of a resistive network model implemented in Matlab. CDEGS is initially used to investigate the self and mutual resistances of metallic structures in various soil structures taking into consideration factors like buried depth, material of conductor, coating, radius, length and geometrical arrangement.

The information provided by CDEGS is then appropriately employed in a simplified resistive network that speeds up the simulation time while at the same time maintains the accuracy of the results produced. This form of model has a number of advantages, one of them being the fact that it can be utilized dynamically to acquire a true visualization of the stray current corrosion risk. Figure 2 illustrates the resistive type network. The components of this model are described in more detail in the next section of this paper.

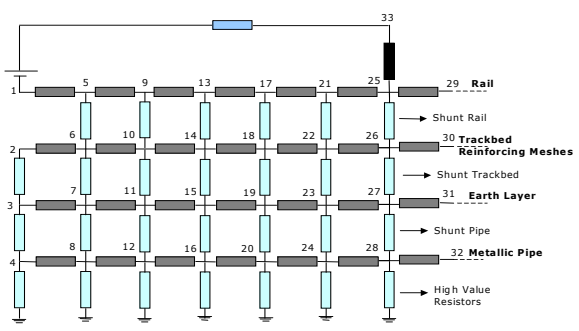

Fig. 2. Resistive network for the rail, the trackbed and the metallic pipe, Floating System.

\section{B. Development Of Parallel Track Model}

The model described has been further enhanced to simulate two parallel tracks that are occupied by two trains; one on each track. This type of modeling constitutes a more realistic case study that can investigate the performance of a DC light rail system and the impact of stray current on supporting and third party infrastructure. With this form of modeling, parameters such as the effect of cross bonding the tracks on the stray current level produced by the system can be examined. In DC electrified railways it is a common practice for the rails of each track to be bonded together and for the tracks to be crossbonded in order to reduce the resistance of the return path, thus reducing the generation of stray currents.

As in previous modeling efforts, geometrically accurate models are first built in the CDEGS software. These models, as shown in Figure 3, are relatively short in length owing to the high complexity of the model. They are used to develop and validate [8] the resistive type network shown in Figure 4. If changes in soil resistivity occur over a system length, multiple CDEGS models would be run to produce different resistive network parameters for each individual case.

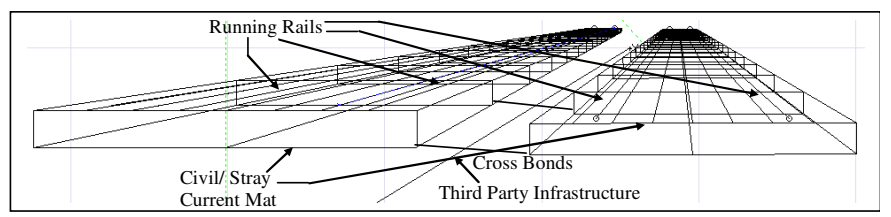

Fig. 3. Geometrically Accurate CDEGS model for two tracks in perspective view

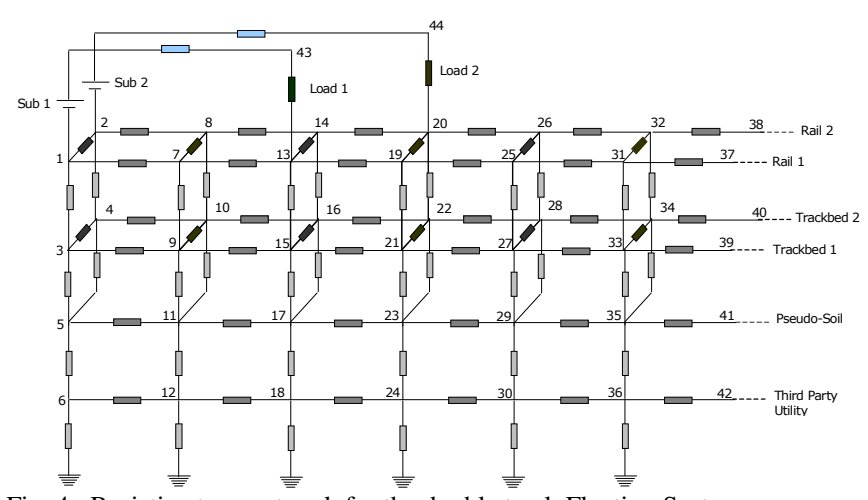

Fig. 4. Resistive type network for the double track Floating System.

The resistive network that has been produced is shown in Figure 4 and includes at the top, two sets of resistors that represent the two tracks. For modeling purposes the equivalent series resistance of each track is represented by one resistor layer based on the assumption that only one rail is used for return current (the other is used for signaling). A shunt resistor then represents the resistance of the rail to the trackbed (in reality this is practically the same as the resistance to earth of the rail for most well coated systems). Below each set of the 
resistors representing the rails, there also exists a resistor layer that represents the equivalent series and shunt resistance of trackbed (stray current collection mat). Each track has its equivalent trackbed situated beneath it.

The third layer is the 'pseudo-earth layer' which can be modified appropriately for different soil structures. This layer is important to include as it is recognized that some current flows through the earth and that this has a finite conductivity. This layer is placed before any third-party infrastructure as current will always flow from the trackbed into the earth before reaching such infrastructure. The final fourth layer represents the series and shunt resistances of this third party infrastructure/ metallic pipe.

The series resistance of the rail, the metallic mat and the buried pipe can easily be calculated using the cross sectional area, length and resistivity of each conductor's material. The series resistance of the pseudo earth layer, varying according to soil resistivity, is determined by an iterative process (the end result requiring the results from a resistive model to match that of the more complex CDEGS model).

Additionally, the resistive model includes 4 sets of shunt resistors that interconnect the different layers. The role of the shunt resistors in conjunction with the 'pseudo-earth layer' is to provide the conductive path between the different structures, these values vary according to the specified soil structure. Of particular note is that the values of shunt resistance for the rail are determined by approximating the resistance of the insulating pads that support the rail on the trackbed. The values of shunt resistance used for the trackbed and the metallic pipe are initially determined by CDEGS by determining the resistance to earth of each structure. This does not, however, fully reflect the resistance between the individual elements and the values of these are therefore iterated along with the series resistance of the pseudo-earth later to reproduce the CDEGS current distribution accurately in the resistive model.

Of all the parameters detailed in this study, the value of the soil resistivity used is likely to introduce the greatest source of error into the modelling process. The CDEGS software has been shown to be accurate by many researchers across the world and has been extensively verified. The models as described in this paper are simplifications of a real system (for example trackbed reinforcement bar would be too complex to allow the modelling of all conductors) but these simplifications were used after confirming that they did not cause an error in any expected current/voltage of more than $1 \%$ [1].

The error introduced by the soil resistivity relates to the fact that it is both difficult to measure and changes as a function of the season / weather conditions. These statements can be qualified by work carried out by other researchers. Dawalibi et al states that soil resistivity measurements can be in error by as much as $50 \%$ when measurements are taken in proximity to metallic buried structures [9]. For a new light rail system in an urban environment, it is highly unlikely that such buried structures will not be present. Other researchers find that soil resistivities can change in a local environment by a factor of 20 according to the level of moisture present within the soil [10]. Soil near the earth boundary is likely to be significantly affected. It would therefore be prudent in any analysis to consider likely variations in soil resistivity over a year and the influence of buried objects on measurements before carrying out simulations.

The system that has been studied is modeled on the basis of the parameters given in Table I. Two tracks are modeled along with the trackbeds that are placed underneath them. The tracks are crossbonded every $100 \mathrm{~m}$ as is the trackbed itself. Crossbonds have been shown to be beneficial in controlling the levels of stray current on a floating DC light rail system [11].

TABLE I

DESIGN PARAMETERS OF THE SIMULATION MODEL

\begin{tabular}{|l|l|c|}
\hline \multirow{3}{*}{ RAILS } & Series Resistance: & $0.04 \Omega / \mathrm{km}$ \\
\cline { 2 - 3 } & Resistance to earth: & $320 \Omega / \mathrm{km}$ \\
\cline { 2 - 3 } & Radius: & $0.048 \mathrm{~m}$ \\
\cline { 2 - 3 } & Number of conductors: & 1 \\
\cline { 2 - 3 } & Buried at depth: & $0.05 \mathrm{~m}$ \\
\hline \hline \multirow{3}{*}{ METALLIC MATS } & Series Resistance: & $0.08 \Omega / \mathrm{km}$ \\
\cline { 2 - 3 } & Resistivity of Coating: & $180 \Omega . \mathrm{m} /$ thickness: $40 \mathrm{~mm}$ \\
\cline { 2 - 3 } & Radius: & $0.0094 \mathrm{~m}$ \\
\cline { 2 - 3 } & Number of conductors: & 10 longitudinal \\
\cline { 2 - 3 } & Buried at depth: & $0.628 \mathrm{~m}$ \\
\hline \hline \multirow{3}{*}{ PIPES } & Series Resistance: & $0.2845 \mathrm{E}-03 \Omega / \mathrm{km}$ \\
\cline { 2 - 3 } & Radius: & 1 hollow \\
\cline { 2 - 3 } & Number of corductors: & $20.628 \mathrm{~m}$ \\
\hline \hline \multirow{3}{*}{ CROSS BONied at depth: } & $1.437 \mathrm{E}-03 \Omega$ \\
& Series Resistance: & $120 \mathrm{~mm} \mathrm{~m}^{2}$ \\
\cline { 2 - 3 } & Cross Sectional Area: & Every $100 \mathrm{~m}$ \\
\cline { 2 - 3 } & Number of conductors: & $10 \mathrm{~m}$ \\
\hline \hline \multirow{2}{*}{ POWER } & Length: & $1000 \mathrm{~A}$ \\
\hline
\end{tabular}

\section{Validation of Static Simulation Model}

As a starting point, the system is modeled in CDEGS to demonstrate how this software can allow the selection of shunt and series parameters that give good agreement when the stray current distribution between the different metallic structures in investigated.

The models shown in Figures 3 and 4 are as implemented in MATLAB using the resistive model. Equivalent geometrically accurate systems were also developed in CDEGS All of the models used in this section relate to systems placed in a soil resistivity of $100 \Omega \mathrm{m}$. Validation of the shunt/series parameters used later in this paper for the alternative soil models have been carried out but are not presented.

The first case study, i.e. Figure 5 illustrates a $1 \mathrm{~km}$ section of two parallel tracks which represent the geometrically accurate CDEGS model of Figure 3 . These $1 \mathrm{~km}$ sections are representative of a symmetrical $2 \mathrm{~km}$ section of two parallel tracks with two trains at the centre of each track and a substation at the same end of each track drawing constant current of 1000 A each. This static model assumes worst load 
conditions; it thus constitutes a worst-case scenario for modeling the impact of stray current on the rails, the trackbed and the third party utility structure.

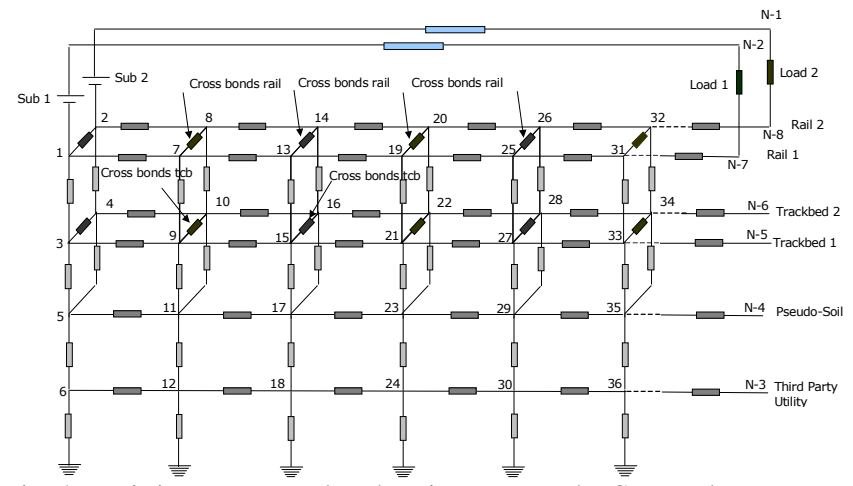

Fig. 5. Resistive type network, substations same end - Case study I.

Figures 6 and 7 illustrate a comparison of the results obtained by CDEGS simulations using the static models presented in Figure 3 and by simulation of the resistive type network (Figure 5) in the case where the tracks are crossbonded. The graphs show the net current leakage profiles of the stray current collection mat (trackbed) and the third party utility. Net current leakage is taken to be the difference between the stray current entering the structure and that leaving the structure (for example on a trackbed, current may enter at the top of the trackbed and some will then exit at the base, the remainder being carried along the trackbed). The maximum difference between the results from the MATLAB resistive model and those obtained by CDEGS is $2.2 \%$. The results are those expected when a floating rail system is simulated, i.e. one half of the system shows current flowing into the metallic conductors while the other half of the system sees current leaving the conductors.

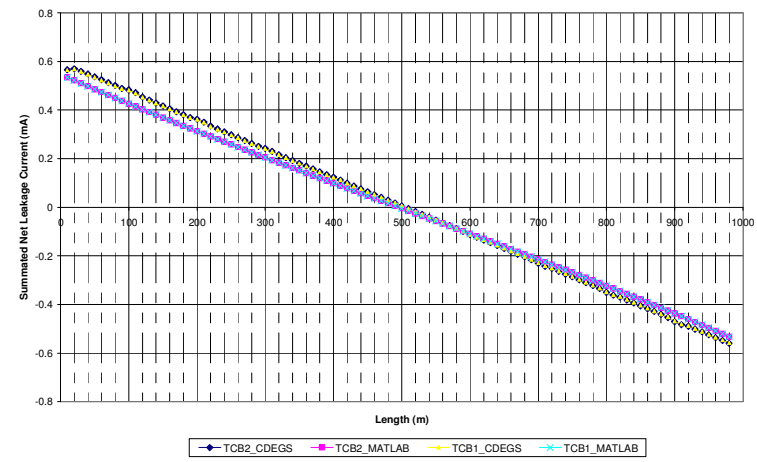

Fig. 6. Summated Net Current Leakage of Trackbeds obtained by CDEGS and MATLAB with the tracks cross-bonded - Case study I

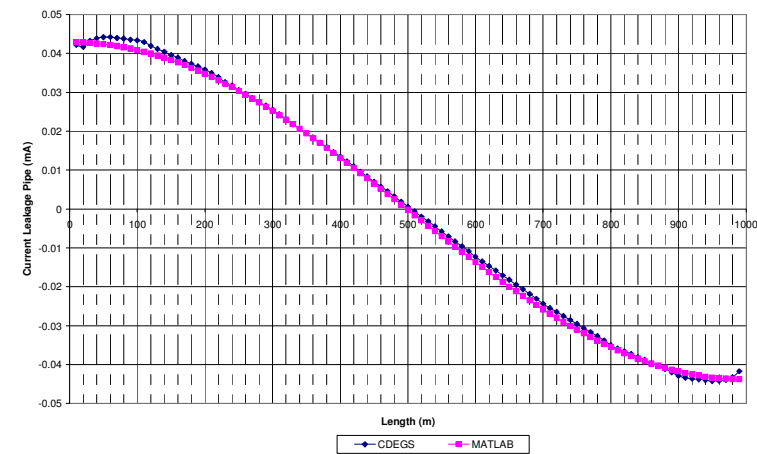

Fig. 7. Summated Net Current Leakage of third party utility obtained by CDEGS and MATLAB with the tracks cross-bonded - Case study I.

The second case study in Figure 8 considers the same basic model used in case study I but has the position of the load (train) and the substation swapped on one of the tracks. The objective of this case study is to demonstrate the effect of substation placement on the stray current level of the rails and the third party infrastructures in addition to the effect of cross bonding the two tracks. The values of the shunt parameters in the resistive network of Figure 8 have not been altered and are the same as those employed in case study Figure 5.

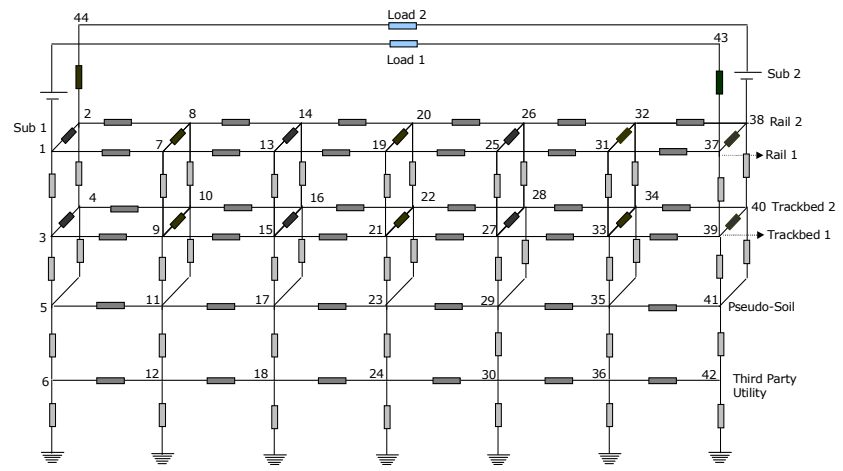

Fig. 8. Resistive type network, substations opposite end- Case study II.

Figures 9 and 10 illustrate a comparison of the results obtained by CDEGS simulations using the models presented in Figure 3 (direction of currents is reversed to cope with the change of substation placement) and by simulation of the resistive type network (Figure 8).

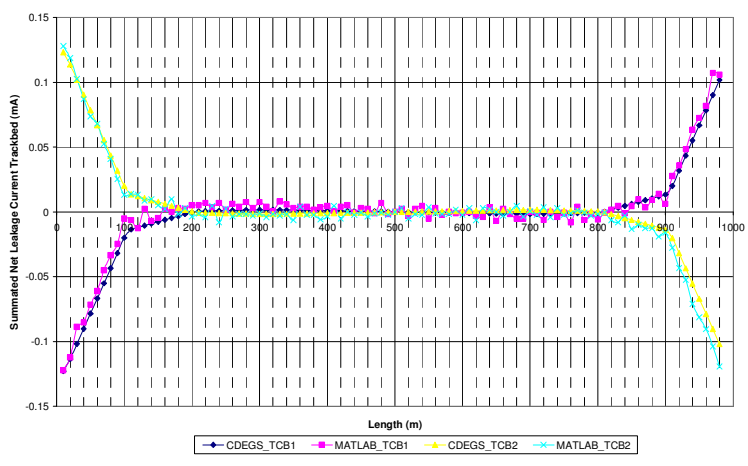

Fig. 9. Summated Net Current Leakage of Trackbeds obtained by CDEGS and MATLAB with the tracks cross-bonded-Case study II 


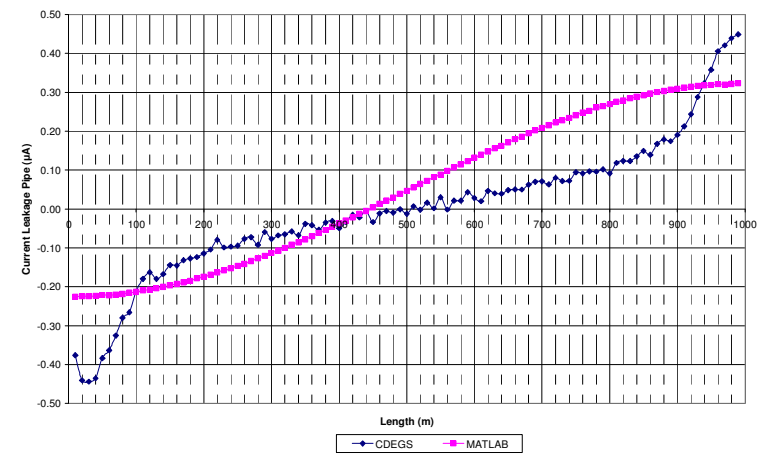

Fig. 10. Summated Net Current Leakage of third party utility obtained by CDEGS and MATLAB with the tracks cross-bonded-Case study II

The results presented in Figure 9 show a good agreement between the Matlab and CDEGS models even though the level of stray current is significantly less, a maximum value of just under $0.15 \mathrm{~mA}$ being observed in comparison to $0.6 \mathrm{~mA}$ in the previous case. The reduction in the level of stray current relates to the flow of traction current in opposite directions and the ultimate cancellation due to the crossbonds connecting the two tracks. The stray current will not be reduced to zero owing to the finite resistance of the track between the crossbond locations and the resistance of the crossbonds themselves.

In the first case, a maximum current slightly lower than $50 \mathrm{uA}$ is seen entering the pipe. In the second case, the simulations show an increased error between the Matlab and CDEGS computations. However, in this case the maximum current seen entering the pipe is less than $5 \mathrm{uA}$ according to CDEGS and just over $2 \mathrm{uA}$ according to Matlab. The discrepancy is attributed to numerical error introduced into the CDEGS results by a matrix inversion process. The error introduced becomes significant at this level of current. However, this level of current is so small that it is not of concern when it is to be used to consider corrosion risk.

The fact that identical series and shunt resistance parameters (for the rails, the trackbeds, the earth layer and the third party utility structure) were employed in both resistive type network models (Figures 5 and 8) and that the results obtained from the developed algorithm in MATLAB and CDEGS modeling are consistent gives confidence in the validity of the proposed simulation tool.

The runtime simulation of the developed algorithm in MATLAB is dramatically reduced when compared with the runtime simulation that an equivalent model in CDEGS requires. This directly relates to the number of elements required to accurately simulate the system and therefore also to the maximum system size that could be simulated (this being driven by memory capacity). This reduction in the simulation runtime and enhancement of the system size that could be studied does not come at the expense of the accuracy of the results.

\section{Investigation Of The Effect Of Alternative Soil Structures}

The purpose of the work is to examine the impact of different soil structures on the stray current distribution. CDEGS allows the user to define the characteristics of the soil in which the conductors are being modeled. If the soil is not uniform, a layered soil model can be specified. A maximum of three horizontal earth layers or three vertical earth layers may be specified in terms of their resistivity and layer thickness. CDEGS could therefore allow the construction of resistive models of systems placed in different soil environments. In terms of implementing this into the resistive model, a system that is considered to pass through three different soil resistivity regions would require three sets of shunt/series parameters from three different CDEGS simulations all using the alternative soil resistivity values.

In this paper, simulations using uniform soils, two layer horizontal soils and two layer-vertical soil resistivity environments are presented utilizing the resistive model simulation tool developed. Ultimately, the simulation tool can be utilized to formulate a soil structure with unlimited variations along the route [7]. Table II gives the specific details of the various models used in this paper.

TABLE II

MODELS EMPLOYED IN SIMULATIONS

\begin{tabular}{|c|c|c|}
\hline \multicolumn{3}{|c|}{ UNIFORM MODEL } \\
\hline Model A & $10 \Omega . m$ & \\
\hline Model B & $1000 \Omega$ s.m & \\
\hline \multicolumn{3}{|c|}{ TWO LAYER HORIZONTAL MODEL } \\
\hline Model C & 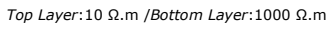 & \\
\hline Model D & 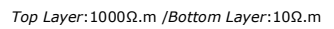 & \\
\hline \multicolumn{3}{|c|}{ TWO LAYER VERTICAL MODEL } \\
\hline Model E & 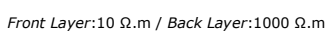 & \\
\hline \multicolumn{3}{|c|}{ FOUR LAYER VERTICAL MODEL } \\
\hline Model F & 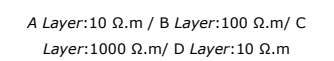 & \\
\hline
\end{tabular}

The uniform models A and B allow all of the systems to be enclosed by the same soil resistivity (although the rails remain coated by a high resistivity layer to enable simulation of the correct rail to earth resistance and the trackbed conductors are coated with a layer equivalent in thickness and resistivity to the cover provided by the trackbed concrete).

In the case of the two-layer horizontal models, the rail and the track bed both lie in the top layer while the metallic pipe lies in the bottom layer. The track bed and rail are placed into the same environment, as they are in close proximity in real DC light rail systems. The two models simulated represent the 
cases where the soil resistivity increases with depth (model C) and the case where the resistivity decreases with depth (model D)

For the two-layer vertical models, the rail, the metallic mat and the pipe all see a change in soil resistivity at a distance of $500 \mathrm{~m}$, i.e. half of their length lies in high resistivity soil whilst the other half lies in low resistivity soil.

For the four-layer vertical models, the rail, the metallic mat and the pipe all see a change in soil resistivity at a distance of $250 \mathrm{~m}$, i.e. a quarter of their length lies in $10 \Omega \mathrm{m}$ resistivity soil, a quarter of their length lies in $100 \Omega$ m resistivity soil, a quarter of their length lies in $1000 \Omega \mathrm{m}$ resistivity soil whilst the last quarter lies in $10 \Omega \mathrm{m}$ resistivity soil.

\section{DynAmic Modelling TeChNIQUES}

True visualization of the impact of the different soil models on the amount of corrosion seen on the transit system and the surrounding infrastructure is only possible using dynamic models. These dynamic models are essentially time-stepped static models in which the train position and velocity varies as a function of distance. In this section, a simple dynamic model has been used in which a train is moved along a 1000m section of track while drawing a constant current (to represent running at constant velocity on a constant gradient). The stray current assessment has been based on an equivalent effective traction current of $1000 \mathrm{~A}$. The results may be scaled to other traction currents if desired, as this is a linear system.

By monitoring the total positive stray current (i.e. current leaking off a metallic structure) during each time step of the simulation, the total corrosive stray current can be obtained by its integration. This is defined as the gross leakage charge. The negative stray current, i.e. that returning onto structures is not considered as this does not cause any corrosion to take place and does not reverse the corrosion process caused by positive stray current leakage.

The simulation results presented in section IV are based on a scenario in which two substations are placed at opposite ends of two tracks and in which the trains are moved in opposite directions as illustrated in Figure 11. The two tracks are crossbonded.

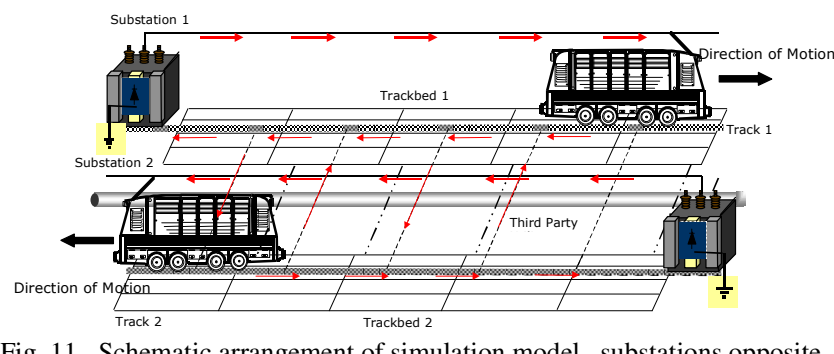

Fig. 11. Schematic arrangement of simulation model-substations opposite end

There is no particular significant in the choice of the system and the results that have been obtained will therefore only serve to give conclusions that relate to the importance of the accurate simulation of soil structures.

Figure 12 illustrates a flowchart of the operation of the software in terms of the calculation of the stray current distribution for each static model and the procedure that then determines the gross leakage charge of the rails, trackbeds and the third party utility structure.

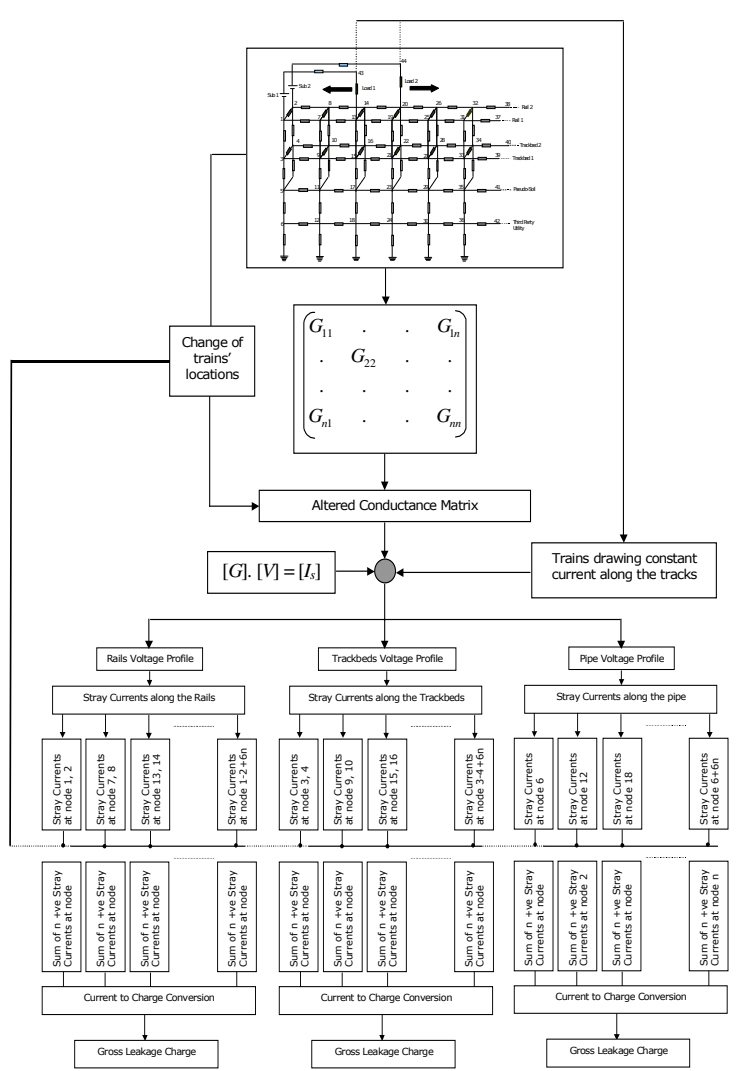

Fig. 12. Flow chart to determine the gross leakage charge

\section{Simulation Results}

When the system is simulated with one movement of the trains, i.e. from one end of the track to the other (respectively), the gross leakage charge for all of the individual system elements can be described. The gross leakage charge of the rail is the same for all soil resistivity models due to the high resistance of the coating layer placed along the rails. Figure 13 illustrates the current leakage density profile for all 3 uniform model cases.

Figure 14 illustrates the corrosive leakage charge of the base of trackbed for all soil resistivity models except $\mathrm{F}$ (this is used later in the paper). Only the base of the trackbed is considered since corrosion at the top of the trackbed is assumed to be proportional to the rail leakage current (owing to the direction in which the current will flow into the trackbed from the rails) and is therefore invariant as a function of soil resistivity. 
Figure 14 shows the significant impact of the vertical soil structure, model $\mathrm{E}$, on the leakage current with a large discontinuity being present at the soil structure boundary. It is significant that in the lowest soil resistivity region $(10 \Omega \mathrm{m}$ at $0 \mathrm{~m}$ to $500 \mathrm{~m}$ ), the leakage currents observed are higher than those seen in soil model A that has a uniform resistivity of $10 \Omega \mathrm{m}$.

When comparing models B \& D, the performance of the trackbed stays reasonably constant even though in $\mathrm{D}$ the bottom layer resistivity has decreased significantly from $1000 \Omega \mathrm{m}$ to $10 \Omega \mathrm{m}$. The reason for the performance of the trackbed staying reasonably constant is the high reflectivity factor at the boundary between the top and bottom soil layers. When the upper layer resistivity is large in comparison to the lower layer as in model $\mathrm{D}$, the reflection index is approximately 1 and current cannot penetrate into the lower layer [9]. Current can therefore not easily reach the pipe or the lower resistivity and is retained on the trackbed in a similar way to the $1000 \Omega$ m uniform soil model.

When comparing models A \& $\mathrm{C}$, the bottom layer resistivity increases from $10 \Omega \mathrm{m}$ to $1000 \Omega \mathrm{m}$ and the level of gross leakage charge on the base of trackbed is seen to decrease significantly [1].

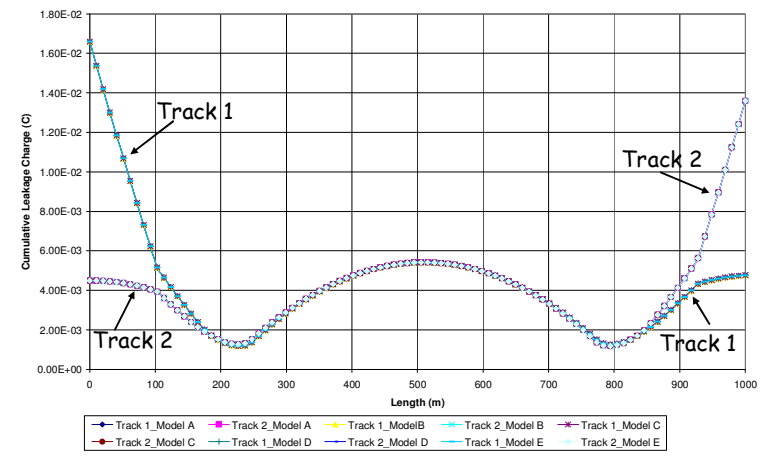

Fig. 13. Cumulative Leakage Charge Rails

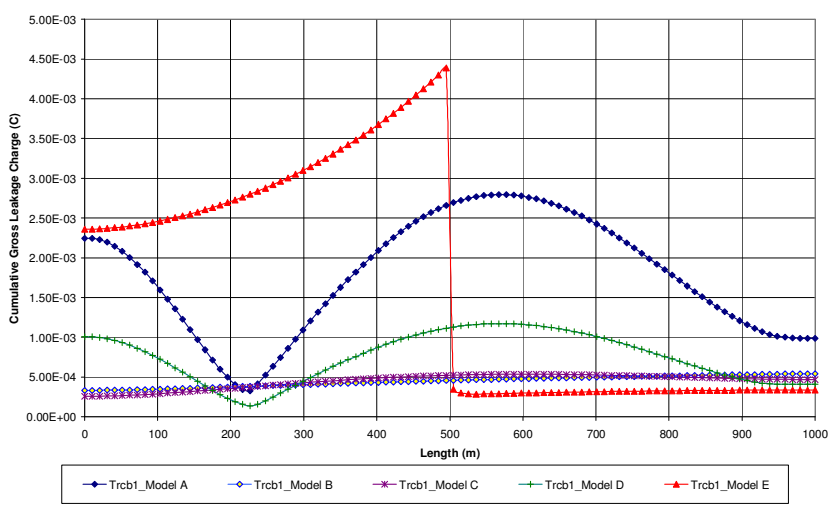

Fig. 14. Cumulative Leakage Charge Base of the Trackbeds

Figure 15 illustrates the cumulative corrosive leakage charge of the third-party utility structure (metallic pipe) for all the soil resistivity models (except F) tabulated in Table II.

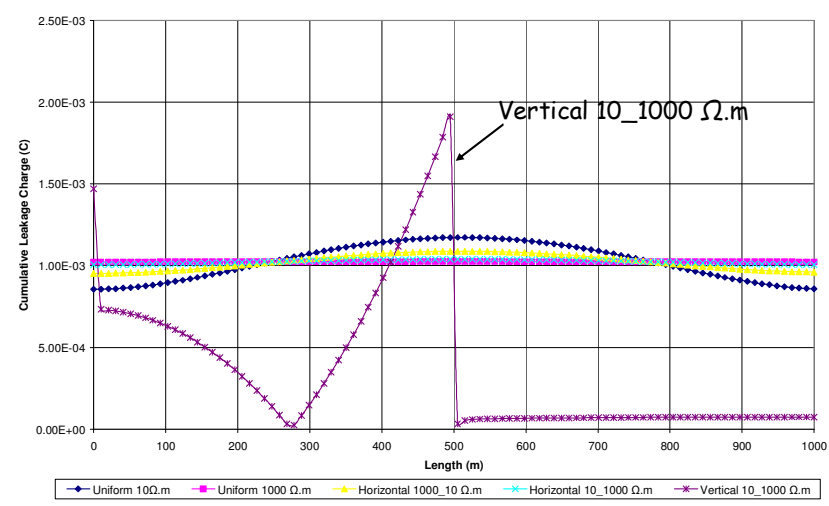

Fig. 15. Cumulative Leakage Charge of third party Infrastructure

The results for the vertical soil model $\mathrm{E}$ verify previous conclusions [1], i.e. that a concentrated current leakage region will exist on the third party utility structure and trackbed when a vertical soil model exists. The discontinuities highlighted in Figure 14 and 15 show that some portions of the system around the soil interfaces are more at risk.

The results shown in Figure 15 also illustrate the fact that in homogenous systems, high soil resistivities mean that thirdparty buried structures are less vulnerable to corrosion damage while in low soil resistivities the converse is true.

\section{EXAMPLE OF MODELLING A REAL SYSTEM}

The work detailed is intended to aid in the accurate modelling of real transit systems. This example of modelling utilises Model F which is a four layer vertical soil structure as illustrated in Table II. Details on the technique utilized to formulate a multi-layer soil structure are given in [8]. An example of the use of the modelling in the analysis of a simple system is now presented.

The model consists of two $1 \mathrm{~km}$ tracks as shown in Figure 11 on which the trains move with a constant velocity in opposite directions and hence draw a constant current. The current used in this simulation is $1000 \mathrm{~A}$. The results could be linearly scaled for other values of current.

The operational condition is that trains move along the section of track with a headway of 3 minutes. This will result in 20 trains running per hour on each track, therefore 40 trains per hour on both tracks. Assuming that services are running for 19 hours i.e. from 5 a.m. until midnight, the total number of trains that will run across the section of two tracks under study would be 760 . Therefore the total charge produced by the movement of 760 trains will be 760 times more than the charge produced by the operation of one simulation run.

The model must assess the cumulative impact of this stray 
current on the rails, the stray current collection system and any surrounding metallic infrastructure.

As an example of the approach taken to convert the values of current from the model to lifetime, 10 interconnected $8 \mathrm{~mm}$ bars are used to form the stray current mat placed under rail. When current leaks onto the mat (from the rail) and off the mat (into the soil), it is assumed that the current will be evenly distributed over the whole mat but only on the half of the bar closest to the interface. This assumption is based on studies of an entire mat within the CDEGS software [1], [8].

For one meter of stray current control mat, the surface area vulnerable to corrosion is therefore:

$$
0.5 \times \pi \times 10 \times 8 \mathrm{~mm} \times 1000 \mathrm{~mm}=125664 \mathrm{~mm}^{2}
$$

The current flowing onto/leaving the mat at a particular location can be converted to a current density using this area and the corrosion rate (for the areas where current is leaving the mat) can be determined using equation (1).

$$
\text { Corrosion_rate }=\frac{\text { Icorr }}{n F}
$$

In equation (1), Icorr is the corrosion current density in $\mathrm{A} / \mathrm{m}^{2}, F$ is Faraday's constant $96,490 \mathrm{C} /$ mole, and $n$ is the number of electrons transferred per molecule of a metal corroded. The corrosion rate is the number of corroded moles of metal per square metre per second, which converts to grams per square meter per day $\left(\mathrm{g} / \mathrm{m}^{2} / \mathrm{d}\right)$, by multiplying by the atomic weight of the metal.

Figures 16 and 17 show an example of the application of the model and this equation. These graphs give the metal loss that will be observed along the entire length of trackbed bars and on the entire length of metallic pipe in one year, for the vertical four-layer soil structure.

For oxygenated areas of the system, the depth of steel corrosion required to cause cracking of the concrete, and thus allow rapid penetration of chloride ions to the steel is typically thought to be in the range of $150 \mu \mathrm{m}-200 \mu \mathrm{m}$. Using this thickness to estimate the life of the system, Figures 16 and 17 also illustrate the corrosion life calculation of a section of a trackbed and of the metallic pipe based on the $150 \mu \mathrm{m}$ threshold, for the vertical four-layer soil structure.

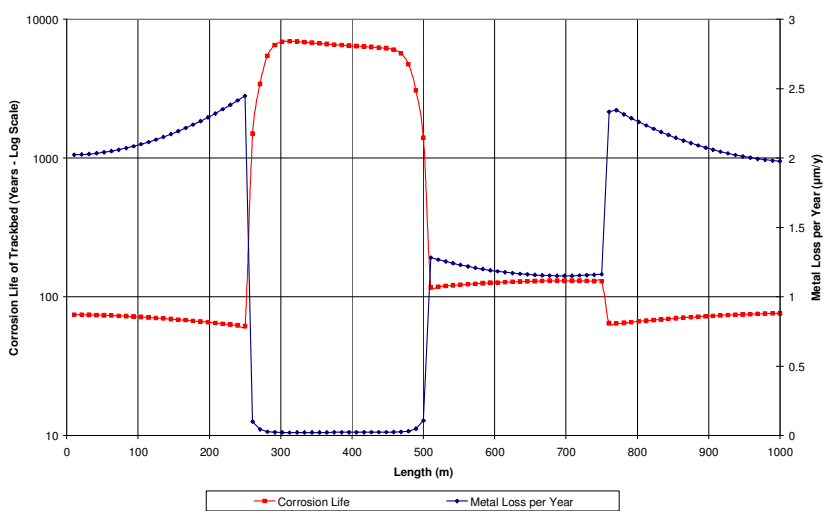

Fig. 16 Metal loss per year and corrosion life of trackbed

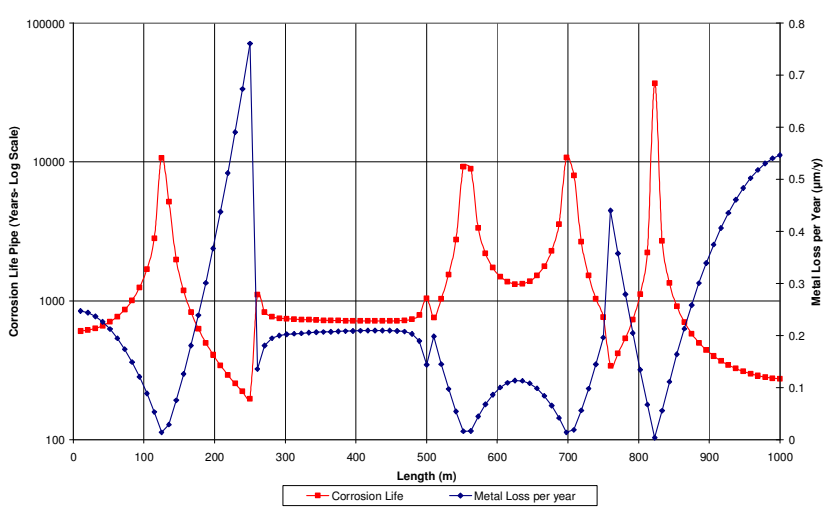

Fig. 17 Metal loss per year and corrosion life of third party infrastructure

Significant peaks of corrosion, corresponding to points with lower lifetimes, can be seen in the system as a result of the soil interfaces. The results seen are not simple to interpret and show the need for the use of a software tool in this form of lifetime prediction. In areas of the system with a lower lifetime, extra stray current management in the form of upgraded rail insulation, larger trackbed conductors or a stray current control cable could be used to give a longer system lifetime. This is the main benefit of this form of modeling approach, the ability to define an economic stray current control system.

\section{CONCLUSIONS}

A simulation model has been developed that employs a resistive networks to solve the stray current distribution found in / around a DC rail system. Data for formulation of the resistive network is provided by CDEGS, a commercial tool capable of simulating geometrically accurate systems (such as the trackbed mat).

The use of the resistive model significantly reduces simulation runtime and CPU memory usage, when compared with an equivalent CDEGS model. This is not at the expense of accuracy. The very important advantage of the developed model is its ability to be simulated dynamically, in contrast with the CDEGS model, which is realistically restricted to non-dynamic case studies within the transit systems. 
Furthermore a four vertical layer soil structure has been modeled (CDEGS is limited to max. three vertical layers). The resistive modeling technique can be utilized to formulate a soil structure with unlimited variations along the route that has no real limitations on length beyond the memory capability of the computer it is being run on.

This paper uses a resistive model to demonstrate the influence different soil models have on the corrosion performance of the rail, trackbed, and a metallic pipe. In uniform soil environment severe corrosion on pipelines predominates in sections where there is a low earth resistivity. Horizontally layered soils where the earth resistivity shows a decrease with depth are dominated by the behaviour of the upper layer. For vertical models, a concentrated current leakage region will exist on the third party utility structure and trackbed at the point where the soil discontinuity occurs. This can give rise to higher stray currents that a system in a uniform soil resistivity with the value of the lowest vertical layer.

The model can be used to optimize the level of stray current protection required along the system length. While this would depend on the provision of accurate soil data, the example used does show how the simulation tool could be used to optimize the stray current control over the length of the system.

\section{ACKNOWLEDGMENT}

The authors gratefully acknowledge the valuable technical and financial contribution of CAPCIS Ltd on the research carried out.

\section{REFERENCES}

[1] C Charalambous, I Cotton 'Influence of Soil Structures on Corrosion Performance of Floating DC Transit Systems', IEE Proceedings Electrical Power Applications, Under Press.

[2] C Charalambous, I Cotton 'Impact of Soil Topologies on Stray Current Performance of a DC Mass Transit System', Proceedings of the XIVth International Symposium on High Voltage Engineering, China, August 25-29, 2005

[3] I Cotton, C Charalambous, P Ernst, P Aylott, 'Stray Current Control in DC Mass Transit Systems', IEEE Transactions Vehicular Technology, Vol.54, No 2, March 2005

[4] [6] Yu J G. "The Effects Of Earthing Strategies On Rail Potential And Stray Currents In DC Transit Railways", Proceedings of The International Conference On Developments In Mass Transit Systems, 20th - 23rd April 1998

[5] Case, S, "DC Traction Stray Current Control - Offer a Stray a Good Ohm?” (Ref. No. 1999/212), IEE Seminar on, 21 Oct. 1999. Pages: 1/1 $-1 / 6$.

[6] J. G. Yu, " The Effects of Earthing Strategies on Rail Potential and Stray Currents in DC Railways", Proc. of IEE International Conference on Developments in Mass Transit Systems, London, UK, pp 303-309, 1998.

[7] CDEGS software, Safe Engineering Services \& Technologies Ltd, Montréal Canada est 1978.

[8] C. Charalambous, Stray Current Control and Corrosion Limitation for DC Mass Transit Systems, Ph.D Thesis, University of Manchester, Sep. 2005.
[9] Ma, J.; Dawalibi, F.P.; Study of influence of buried metallic structures on soil resistivity measurements, Power Delivery, IEEE Transactions on, Volume: 13, Issue: 2 April 1998 Pages:356 - 365.

[10] Calculation of earth resistance for a deep-driven rod in a multi-layer earth structure, Takahashi, T.; Kawase, T.; Power Delivery, IEEE Transactions on, Volume: 6, Issue: 2, April 1991,Pages:608 - 614.

[11] Bahra, K.S.; Catlow, R.B.; Control of stray currents for DC traction systems Electric Railways in a United Europe, 1995, International Conference on 27-30 Mar 1995 Page(s): 136 - 142.

\section{BIOGRAPHIES}

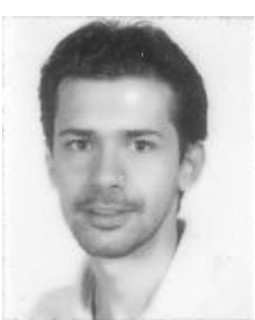

Charalambos Charalambous was born in Nicosia, Cyprus in 1979. He received a Class I BEng (Hons) degree in Electrical \& Electronic Engineering in 2002 and $\mathrm{PhD}$ in Electrical Power Engineering in 2005 from UMIST, UK (The University of Manchester). He is currently a Research Associate within the National Grid High Voltage Research Center in the University of Manchester, UK. His current research interests include low frequency transformer modeling, and the electrical control and analysis of DC corrosion.

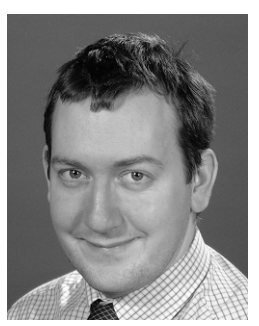

Ian Cotton (M'98) was born in the UK in 1974. He received a Class I BEng (Hons) degree in Electrical Engineering from the University of Sheffield in 1995 and a PhD in Electrical Engineering from UMIST in 1998. He is currently a Lecturer in the Electrical Energy and Power Systems Group of the School Of Electrical and Electronic Engineering at the University of Manchester. His current research interests include power systems transients, the use of higher voltage systems in aerospace applications and power system induced corrosion. He is a Member of the Institution of Electrical Engineers and a Chartered Engineer.

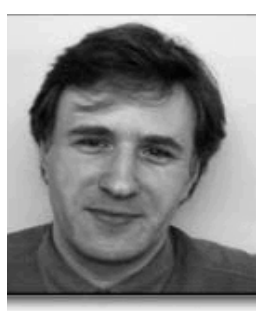

Pete Aylott is a Director of CAPCIS. His main area of work relates to stray current management of rail systems in the UK and overseas. 